\title{
CONTINUITY PROPERTIES OF SOLUTIONS OF MULTIVALUED EQUATIONS WITH WHITE NOISE PERTURBATION
}

\author{
MARIUSZ MICHTA \\ Technical University, Institute of Mathematics \\ Podgorna 50, 65-246 Zielona Gora, Poland
}

(Received April, 1996; Revised November, 1996)

\begin{abstract}
In the paper, we consider a set-valued stochastic equation with stochastic perturbation in a Banach space. We prove first the existence theorem and then study continuity properties of solutions.
\end{abstract}

Key words: Set-valued Mappings, Aumann's Integral, Convergence in Probability of Random Elements.

AMS subject classifications: $54 \mathrm{C} 65,54 \mathrm{C} 60$.

\section{Preliminaries}

Problems of existence of solutions to set-valued differential equations were studied by many (see e.g., $[3,8,9]$ ). In particular, random cases were considered by the author in $[11,12]$.

In this paper we study the set-valued stochastic equation with white noise drift:

$$
\begin{gathered}
D X_{t}=F\left(t, X_{t}\right) d t+\sigma_{t} d w_{t}, t \in I, \\
X_{0}=U \quad P .1
\end{gathered}
$$

where $F$ and $U$ are given random set-valued mappings with values in the space $K_{c}(E)$, of all nonempty, compact and convex subsets of the separable Banach space $(E,\|\|), I:=[0, T] ; T>0$. We assume also that there is a predictable stochastic process $\sigma$ with values in $E$. Finally, $\left(w_{t}\right)_{t \in I}$ denotes a real Wiener process. We interpret the above equation through its integral form as

$$
X_{t}=U+\int_{0}^{t} F\left(s, X_{s}\right) d s+\int_{0}^{t} \sigma_{s} d w_{s} \quad P .1, \quad t \in I .
$$

Integrals above are Aumann's integral of $F$ and stochastic (Itô) integral of $\sigma$, respectively.

The aim of this work is to study continuity properties of set-valued solutions of 
$(I)$. First, we recall several notions needed in the sequel. In the space $K_{c}(E)$ we consider the Hausdorff metric $H$ (see e.g., [5, 7]): $H(A, B)=\max (\bar{H}(A, B), \stackrel{c}{H}(B, A))$ for $A, B \in K_{c}(E)$, where $\bar{H}(A, B)=\operatorname{supinf}_{a \in A}\|a-b\|$. By $\|A\|$ we denote the distance $H(A, 0)$. It can be proved that $\left(K_{c}(E), H\right)$ is a Polish metric space.

By $C_{I}=C\left(I, K_{c}(E)\right)$ we denote the space of all $H$-continuous set-valued mappings. In this space we consider metric $\rho$ of uniform convergence:

$$
\rho(X, Y):=\sup _{0 \leq t \leq T} H(X(t), Y(t)), \text { for } X, Y \in C_{I} .
$$

Then we have a Polish metric space.

Let $\left(\Omega, \mathcal{F}_{,} \mathcal{F}_{t}, P\right)_{t \in I}$ be a given complete filtered probability space satisfying the usual conditions. We recall the notion of a multivalued $\mathscr{F}_{t}$-adapted stochastic process. The family of set-valued mappings $X=\left(X_{t}\right)_{t \in I}$ is said to be a multivalued

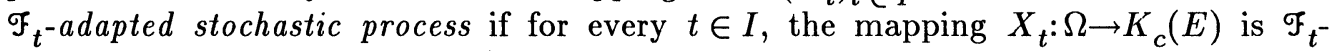
measurable, i.e., $\left\{\omega: X_{t} \cap V \neq \emptyset\right\} \in \mathcal{F}_{t}$, for every open set $V \subset E$ (see e.g., [7]). It can be noted that $V$ can be chosen as a closed or Borel subset. If the mapping $t \rightarrow X_{t}(\omega)$ is $H$-continuous with probability one $(P .1)$ then we say it has continuous paths. In this case, the set-valued process $X$ can be thought as random element $X: \Omega \rightarrow C_{I}$. Let $\left(X_{n}\right)$ be a sequence of random elements with values in metric space $(S, \rho)$. Then we say that $X_{n}$ converges in probability to the random element $X: \Omega \rightarrow S\left(X_{n} \stackrel{P}{\rightarrow}\right)$, of for every $\epsilon>0$, it holds true that $P\left(\rho\left(X_{n}, X\right) \geq \epsilon\right) \rightarrow 0$, as $n$ tends to infinity. It is known (see e.g., [13]) that $X_{n} \stackrel{P}{\rightarrow} X$ if and only if every subsequence of $\left(X_{n}\right)$ has a subsequence converging to $X$ with probability one (P.1).

In the theory of differential equations in Banach space the notion of measure of noncompactness plays one of the central roles (see e.g., [1]). Let $B(E)$ denote a family of all nonempty and bounded subsets in $E$.

Definition 1: The mapping $\mathcal{N}: B(E) \rightarrow[0, \infty)$, defined by $\mathcal{N}(A)=\inf \{\epsilon>0: A$ can be covered with a finite number of balls of radii $\leq \epsilon\}$, is called Hausdorff (ball) measure of noncompactness.

\section{A Set-Valued Stochastic Equation and Stochastic Inclusion}

We begin with the designation of restrictions imposed on $F, U$ and $\sigma$. Let us assume that $F: I \times \Omega \times K_{c}(E) \rightarrow K_{c}(E), \quad U: \Omega \rightarrow K_{c}(E)$, and $\sigma: I \times \Omega \rightarrow E$ have the following properties:

1) $\quad F$ is an integrably bounded multifunction i.e. there exists a joint measurable function $m: I \times \Omega \rightarrow R_{+}$such that $\int_{0}^{T} m(s, \omega) d s<\infty P .1$ and $\|F(t, \omega, A)\| \leq m(t, \omega) P .1, t$-a.e. $A \in K_{c}(E)$.

2) $\quad F(t, \omega$,$) is H$-continuous with $P .1, t$-a.e.

3) $\quad F(t,, A)$ is $\mathscr{F}_{t}$-adapted for every $t \in I, A \in K_{c}(E)$.

4) $\quad F(,, A)$ is measurable for every $A \in K_{c}(E)$.

5) $U$ is an $\mathscr{F}_{0}$-measurable multifunction.
$\sigma=\left(\sigma_{t}\right)$ is an $\mathscr{F}_{t}$-adapted stochastic process for which $E \int_{0}^{T}\left\|\sigma_{s}\right\|^{2} d s$ is
finite.

Let us notice that under assumptions given above, for every $A \in K_{c}(E)$, the setvalued process 


$$
\Phi_{t}=U+\int_{0}^{t} F(s, A) d s+\int_{0}^{t} \sigma_{s} d w_{s}, t \in I,
$$

is $\mathcal{F}_{t}$-adapted with values in $K_{c}(E)$. It is also clear that $\Phi$ has continuous "paths".

We also assume the so-called "Kamke condition" imposed on multifunction $F$ : for every $A_{1}, A_{2}, \ldots \in K_{c}(E)$ one has

$$
\mathcal{N}\left(\bigcup_{n \geq 1} F\left(t, A_{n}\right)\right) \leq k\left(t, \mathcal{N}\left(\bigcup_{n \geq 1} A_{n}\right)\right) \text { with } P .1 \quad t \in I \text { a.e. }
$$

where $k: I \times \Omega \times R \rightarrow R+$ satisfies the following conditions:

a) $\quad k(t,, x)$ is $\mathcal{F}_{-}$-measurable for every $(t, x) \in I \times R_{+}$,

b) $\quad k(, \omega$,$) is a Kamke function (see e.g., [14]) with P.1.$

Definition 2: A multivalued process $X=\left(X_{t}\right)_{t \in I}$ is said to be a solution of $(I)$ if it satisfies multivalued stochastic equation $(I I)$.

Let us notice that without stochastic perturbation, equation $(I I)$ can be written as:

$$
\begin{gathered}
D_{H} X_{t}=F\left(t, X_{t}\right) P .1, \quad t \text {-a.e. } \\
X_{0}=U P .1
\end{gathered}
$$

where $D_{H}$ denotes the Hukuchara derivative operator ([6]) for multifunctions.

Before stating the existence theorem to equation (II) let us recall its special case.

Theorem 1: ([11]) Let $F$ and $U$ be multivalued mappings satisfying conditions 1)4) and 5), respectively. Let us also suppose that $F$ satisfies the "Kamke condition." Then the multivalued random differential equation

$$
\begin{gathered}
D_{H} X_{t}=F\left(t, X_{t}\right) \text { with P.1 } t \in I \text { a.e. } \\
X_{0}=U \text { with P.1 }
\end{gathered}
$$

has at least one solution.

Remark: In fact, the existence of solutions to the above initial value problem is based on the fact that under these conditions there exists at least one solution to the multivalued equation $X_{t}=U+\int_{0}^{t} F\left(s, X_{s}\right) d s$ and on well-known connection between Aumann's integral of set-valued mapping and its Hukuchara derivative via Radström Embedding Theorem (see e.g. [14]).

Theorem 2: Let $E$ be a Banach space such that its dual $E^{*}$ is separable. If $F, U$ and $\sigma$ have properties 1)-6) and F satisfies the "Kamke condition" then there exists at least one solution of the equation (II).

Proof: Let $\xi_{t}=\int_{0}^{t} \sigma_{s} d w_{s}$. Let $\hat{X_{t}}$ : $=X_{t}-\xi_{t}$, where $X_{t}$ is a solution of $(I I)$, and

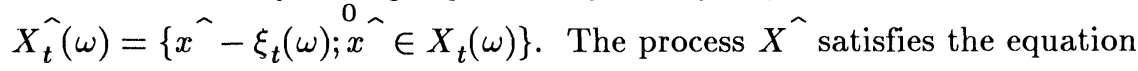

$$
\widehat{X_{t}}=U+\int_{0}^{t} \hat{F^{(}}\left(s, \hat{X_{s}}\right) d s \quad P .1, t \in I,
$$

where $\hat{F}(s, \omega, A)=F\left(s, \omega, A+\xi_{s}(\omega)\right)$. The set-valued mapping $\widehat{F^{-}}$meets properties 
1)-4). By properties of measure of noncompactness it also satisfies (*) (cf. [1]). Hence, equation $(I I)$ has at least one solution if and only if equation (**) has one. By Theorem 1 (via Remark 1) the proof is completed.

Let us suppose now that $\Gamma: I \times \Omega \times E \rightarrow K_{c}(E)$ is a given set-valued mapping. Let us set $F(t, \omega, A):=\overline{c o} \Gamma(t, \omega, A), A \in K_{c}(E)$, where $\overline{c o} B$ denotes the closed convex hull of the set $B$. It is noteworthy to observe the connections between solutions of equation $(I I)$, with $F=\overline{\mathrm{co}} \Gamma$ and solutions of stochastic inclusion

$$
\begin{gathered}
x_{t}-x_{s} \in \int_{s}^{t} \Gamma\left(u, x_{u}\right) d u+\int_{s}^{t} \sigma_{u} d w_{u} \text { with } P .1,0 \leq s \leq t \leq T \\
x_{0} \in U \text { with } P .1 .
\end{gathered}
$$

We suppose that $\Gamma$ is an integrable bounded multifunction such that:

$\left.1^{\prime}\right) \quad \Gamma(t, \omega$,$) is H$-continuous with $P .1, t$-a.e.,

$\left.2^{\prime}\right) \quad \Gamma(t,, x)$ is $\mathcal{F}_{t}$-adapted for every $t \in I, x \in E$,

$\left.3^{\prime}\right) \quad \Gamma(,, x)$ is measurable for every $x \in E$,

$\left.4^{\prime}\right) \quad \forall A \subset S_{r}(U): \mathcal{N}(\Gamma(t, A)) \leq k(t, \mathcal{N}(A)) P .1, t \in I$,

where $S_{r}(U)=U+r B(0,1)$ and $B(0,1)$ is a closed unit ball in Banach space $E$, centered at zero.

Theorem 3: Suppose that $\Gamma$ satisfies conditions $1^{\prime}-4^{\prime}$. If a multivalued stochastic process $X=\left(X_{t}\right)_{t \in I}$ is a solution of equation $(I I)$ with $F=\overline{c o} \Gamma$ then there exists stochastic process $x=\left(x_{t}\right)$ being both a solution to stochastic inclusion $\left(I I^{\prime}\right)$ and the

selection of $X$.
Proof: Similarly, as above, let $\xi_{t}=\int_{0}^{t} \sigma_{s} d w_{s}, \widehat{\Gamma}(t, \omega, x):=\Gamma\left(t, \omega, x+\xi_{t}(\omega)\right)$ and

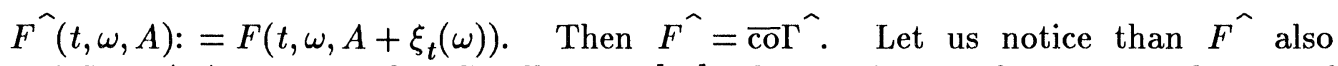
satisfies $1^{\prime}-4^{\prime}$. Hence, by Corollary 1 [11], there exists at least one solution of equation

$$
\hat{X_{t}}=U+\int_{0}^{t} \hat{F^{(}}\left(s, \hat{X_{s}}\right) d s \quad P .1, t \in I .
$$

Taking $X=X^{\widehat{ }}+\xi$ we get a solution of equation $(I I)$, where $F=\overline{c o} \Gamma$. Moreover, by Theorem 4 [11] there exists stochastic process, say $x=\left(x_{t}\right)$, being a selection of $\widehat{X}$ such that: $\widehat{x_{t}}-\widehat{x_{s}} \in \int_{s}^{t} \widehat{\Gamma^{-}}\left(u, \widehat{x_{u}}\right) d u$ with $P .1,0 \leq s \leq t \leq T$, and $\hat{x_{0}} \in U$ P.1.

Consequently, there exists stochastic process $x=\left(x_{t}\right)$, as a selection of $X$, such that: $\widehat{x_{t}}=x_{t}-\xi_{t}$ with P.1. It remains to observe that $x$ is a desired solution of inclusion $\left(I I^{\prime}\right)$.

\section{Continuity Properties of Solutions}

By $S(I \times \Omega)$ we denote the class of "simple" multivalued processes that can be expressed by: $X=\sum_{i=1}^{n} I_{D_{i}} C_{i}$, where the sets $D_{i}, i=1,2, \ldots, n$ form a measurable partition of $I \times \Omega$ and $C_{i} \in K_{c}(E), i=1,2, \ldots, n$.

Lemma 1: If $X=\left(X_{t}\right)_{t \in T}$ is a multivalued stochastic process with continuous 
"paths" then there exists a sequence $\left\{X_{n}\right\} \subseteq S(I \times \Omega)$ such that $\forall(t, \omega) \in I \times \Omega$ : $\lim _{n \rightarrow \infty} H\left(X(t, \omega), X_{n}(t, \omega)\right)=0$.

Proof: It follows directly from the fact that $K_{c}(E)$ is a separable metric space and Proposition 1.9 [15].

Let $\Lambda$ be a metric space. Let us consider the multivalued mapping $F: I \times \Omega \times$ $K_{c}(E) \times \Lambda \rightarrow K_{c}(E)$ such that:

A1. For every fixed $A \in K_{c}(E)$ and $\lambda \in \Lambda, F(,, A, \lambda)$ is a measurable and integrably bounded multifunction.

A2. The mapping $F(t, \omega,, \lambda)$ is with $P .1$ uniformly continuous with respect to $t \in I$ and $\lambda \in \Lambda$.

Definition 2: A multifunction $F$ (with properties A1 and A2) is said to be integrably continuous in probability (icp) at $\lambda_{0} \in \Lambda$ with respect to a family $\mathrm{e} \subset K_{c}(E)(e \neq \emptyset)$ if

$$
\forall C \in \mathcal{C}, \forall t \in I: \quad \int_{0}^{t} F(s, C, \lambda) d s \stackrel{P}{\rightarrow} \int_{0}^{t} F\left(s, C, \lambda_{0}\right) d s
$$

for $\lambda \rightarrow \lambda_{0}$.

The results presented below give characterizations of icp multifunctions. We use them to obtain the main theorem.

Lemma 2: If $F$ is an icp multifunction at $\lambda_{0}$ with respect to $C$ then for every $C \in \mathrm{C}$ one has: $\int_{0}^{t} F\left(s, C, \lambda_{n}\right) d s \rightarrow \int_{0}^{t} F\left(s, C, \lambda_{0}\right) d s$ P.1 uniformly in $t \in I$, for some sequence $\left(\lambda_{n}\right)$ convergent to $\lambda_{0}$.

Proof: Let $D$ be a set of rationals in $I, D=\left\{t_{1}, t_{2}, \ldots\right\}$ and let $\left(\lambda_{n}\right)$ be an arbitrary sequence of elements of $\Lambda$ that converges to $\lambda_{0}$. Fix $C \in \mathcal{C}$. Then for $t_{1} \in D$, there exist a sequence $\left(\lambda_{n}\left(t_{1}\right)\right)_{n}$, convergent to $\lambda_{0}$ and set $\Omega\left(t_{1}\right) \subset \Omega$, $P\left(\Omega\left(t_{1}\right)\right)=1$, such that

$$
\forall \omega \in \Omega\left(t_{1}\right): H\left(\int_{0}^{t_{1}} F\left(s, \omega, C, \lambda_{n}\left(t_{1}\right)\right) d s, \int_{0}^{t_{1}} F\left(s, \omega, C, \lambda_{0}\right) d s\right) \rightarrow 0 \text {, for } n \rightarrow \infty .
$$

Similarly, for $t_{2} \in D$ we can find a sequence $\left(\lambda_{n}\left(t_{2}\right)\right)_{n}$ being a subsequence of $\left(\lambda_{n}\left(t_{1}\right)\right)_{n}$ and $\Omega\left(t_{2}\right) \subset \Omega, P\left(\Omega\left(t_{2}\right)\right)=1$ for which a similar convergence holds. Continuing this selection process we obtain the infinite table

$$
\begin{aligned}
& \lambda_{1}\left(t_{1}\right) \lambda_{2}\left(t_{1}\right) \quad \ldots \quad \lambda_{n}\left(t_{1}\right) \quad \ldots \\
& \begin{array}{lllll}
\lambda_{1}\left(t_{2}\right) & \lambda_{2}\left(t_{2}\right) & \ldots & \lambda_{n}\left(t_{2}\right) & \ldots
\end{array} \\
& \lambda_{1}\left(t_{n}\right) \lambda_{2}\left(t_{n}\right) \quad \ldots \quad \lambda_{n}\left(t_{n}\right) \quad \ldots
\end{aligned}
$$

By diagonal selection we can find a sequence $\left(\lambda_{n}\right)_{n}$ being a subsequence of each row of table (1) that converges to $\lambda_{0}$. Let $\Omega_{0}=\bigcap\left\{\Omega\left(t_{n}\right) ; n \geq 1\right\}$. Then $P\left(\Omega_{0}\right)=1$. Moreover,

$$
\forall \omega \in \Omega_{0}, \forall t \in D: H\left(\int_{0}^{t} F\left(s, \omega, C, \lambda_{n}\right) d s, \int_{0}^{t} F\left(s, \omega, C, \lambda_{0}\right) d s\right) \rightarrow 0, n \rightarrow \infty .
$$


Since the set-valued process $J_{t}=\int_{0}^{t} F(s, C, \lambda) d s, t \in I$ has with $P .1$ uniformly continuous "paths", we can find $\Omega_{0}^{\prime}, P\left(\Omega_{0}^{\prime}\right)=1$ such that

This completes the proof.

$\forall \omega \in \Omega_{0}^{\prime}: \sup _{t \in I} H\left(\int_{0}^{t} F\left(s, \omega, C, \lambda_{n}\right) d s, \int_{0}^{t} F\left(s, \omega, C, \lambda_{0}\right) d s\right) \rightarrow 0$, if $n \rightarrow \infty$.

By $\mathbb{B}_{I}$ we denote the $\sigma$-field of Borel subsets of $I$.

Lemma 3: A multifunction $F$ is icp at $\lambda_{0}$ with respect to family $\mathcal{C}$ if and only if: $\forall C \in \mathcal{C}, \forall\left(\lambda_{n}\right) \subset \Lambda: \lambda_{n} \rightarrow \lambda_{0}, \exists\left(\lambda_{n}^{\prime}\right) \subset\left(\lambda_{n}\right):$

$$
H\left(\int_{B} F\left(s, C, \lambda_{n}^{\prime}\right) d s, \int_{B} F\left(s, C, \lambda_{0} d s\right) \rightarrow 0 \quad P .1\right.
$$

as $n \rightarrow \infty$, for every $B \in \mathbb{B}_{I}$.

Proof: Fix $C \in \mathrm{C}$ and let $\left(\lambda_{n}\right)$ be an arbitrary sequence convergent to $\lambda_{0}$. Then by Lemma 2 , we can find its subsequence $\left(\lambda_{n}^{\prime}\right)$ and $\Omega_{0}: P\left(\Omega_{0}\right)=1$ such that for every $\omega \in \Omega_{0}$ and $0 \leq s<t \leq T$,

$$
\int_{s}^{t} F\left(u, \omega, C, \lambda_{n}^{\prime}\right) d u \rightarrow \int_{s}^{t} F\left(u, \omega, C, \lambda_{0}\right) d u, \text { as } n \rightarrow \infty .
$$

Let $\varphi_{:}=\{[s, t): 0 \leq s<t \leq T\}$ and

$$
\mathcal{A}:=\left\{\bigcup_{i=1}^{n} R_{i}: R_{i} \in \mathcal{\varphi}, R_{i} \cap R_{j}=\emptyset, i \neq j, i, j=1,2, \ldots, n, n \geq 1\right\} .
$$

Since $\sigma(\varphi)=\sigma(\mathcal{A})=\mathbb{B}_{I}$ and $\mathcal{A}$ is a ring of subsets of $I$, then for every $\epsilon>0$ and $B \in \mathbb{B}_{I}$, there exists $A \in \mathcal{A}$ such that $|B \Delta A|<\epsilon$ (c.f. e.g., Th. 11.4 [2]), where | | is Lebesgue measure and $B \Delta A:=(B \backslash A) \cup(A \backslash B)$. By integrably boundness of $F$ we get:

$$
\begin{gathered}
H\left(\int_{B} F\left(s, C, \lambda_{n}^{\prime}\right) d s, \int_{B} F\left(s, C, \lambda_{0}\right) d s\right) \leq H\left(\int_{A} F\left(s, C, \lambda_{n}^{\prime}\right) d s, \int_{A} F\left(s, C, \lambda_{0}\right) d s\right) \\
\quad+\int_{B \Delta A} m(s, \omega) d s, \text { for every } A \in \mathcal{A} .
\end{gathered}
$$

Then by (3), $\limsup _{n \rightarrow \infty} H\left(\int_{B} F\left(s, C, \lambda_{n}^{\prime}\right) d s, \int_{B} F\left(s, C, \lambda_{0}\right) d s\right) \leq \int_{B \Delta A} m(s, \omega) d s$.

Taking $A$ sufficiently close to $B$ we claim (2). The converse is obvious.

Lemma 4: A multifunction $F$ is icp at $\lambda_{0}$ with respect to $K_{c}(E)$ if and only if $F$ is icp at $\lambda_{0}$ with respect to $S(I \times \Omega)$.

Proof: Let us assume that $F$ is icp with respect to $K_{c}(E)$. Let $X \in S(I \times \Omega)$. Then there exist $C_{1}, C_{2}, \ldots, C_{r} \in K_{c}(E)$ and a measurable partition $\left\{D_{1}, D_{2}, \ldots, D_{r}\right\}$ of space $I \times \Omega$ such that $X=\sum_{i=1}^{r} I_{D_{i}} C_{i}$. Take $C_{1}$ and $\left(\lambda_{n}\right)$ to be an arbitrary sequence convergent to $\lambda_{0}$. Next let $\left(\lambda_{n_{k}}\right)$ be any subsequence of $\left(\lambda_{n}\right)$. By Lemma 3 there exists a sequence $\left(\lambda_{n, 1}^{\prime}\right)$ being a subsequence of $\left(\lambda_{n_{k}}\right)$ and a subset $\Omega_{0,1} \subseteq \Omega$; 
$P\left(\Omega_{0,1}\right)=1$ such that:

$$
\forall \omega \in \Omega_{0,1}, \forall B \in \mathbb{B}_{I}: \lim _{n \rightarrow \infty} H\left(\int_{B} F\left(s, \omega, C_{1}, \lambda_{n, 1}^{\prime}\right) d s, \int_{B} F\left(s, \omega, C_{1}, \lambda_{0}\right) d s\right)=0 .
$$

Similarly, for $C_{2}$ we can extract a subsequence $\left(\lambda_{n, 2}^{\prime}\right)$ from $\left(\lambda_{n, 1}^{\prime}\right)$ and $\Omega_{0,2} \subseteq \Omega$ $P\left(\Omega_{0,2}\right)=1$, with the desired property, and so on. Thus we obtain a sequence $\left(\lambda_{n, r}^{\prime}\right)$ which is a subsequence of $\left(\lambda_{n, i}^{\prime}\right), i=1,2, \ldots, r-1$ and $\Omega_{0, r}, P\left(\Omega_{0, r}\right)=1$, such that

$$
\forall \omega \in \Omega_{0, r}, \forall B \in \mathbb{B}_{I}: \lim _{n \rightarrow \infty}\left(\int_{B} F\left(s, \omega, C_{r}, \lambda_{n, r}^{\prime}\right) d s, \int_{B} F\left(s, \omega, C_{r}, \lambda_{0}\right) d s\right)=0 .
$$

Let $\Omega_{0}=\bigcap_{1 \leq i \leq r} \Omega_{0,1}$. For any $A \in \mathbb{B}_{I} \otimes \mathcal{F}$ and $\omega \in \Omega$, we define the set $(A)_{\omega}:=$ $\left\{t \in I:(t, \omega) \in{ }^{1} \in{ }^{\prime} A\right\}$. Then $(A)_{\omega} \in \mathbb{B}_{I}$. Let $\omega \in \Omega_{0}$. Then $X(\cdot, \omega)=\sum_{i=1}^{r} I_{\left(D_{i}\right)_{\omega}}(\cdot) C_{1}$ and $\left\{\left(D_{i}\right)_{\omega}: i=1,2, \ldots, r\right\}$ is measurable partition of $I$. Hence, the following inequality holds:

$$
\begin{gathered}
H\left(\int_{0}^{t} F\left(s, \omega, X_{s}, \lambda_{n, r}^{\prime}\right) d s, \int_{0}^{t} F\left(s, \omega, X_{s}, \lambda_{0}\right) d s\right) \\
\leq \sum_{i=1}^{r} H\left(\int_{\left(D_{i}\right)_{\omega} \cap[0, t]} F\left(s, \omega, C_{i}, \lambda_{n, r}^{\prime}\right) d s, \int_{\left(D_{i}\right)_{\omega} \cap[0, t]} F\left(s, \omega, C_{i}, \lambda_{0} d s\right)\right) .
\end{gathered}
$$

It remains to observe that each term of the above sum converges to zero as $n$ tends to infinity.

The converse statement is obvious. It is enough to take $X:=I_{I \times \Omega} C$, for $C \in K_{c}(E)$. This completes the proof.

By $X^{\lambda}$ we denote a multivalued process being the solution of the equation

$$
X_{t}=U+\int_{0}^{t} F\left(s, X_{s}, \lambda\right) d s+\int_{0}^{t} \sigma_{s} d w_{s} P .1, t \in I, \lambda \in A .
$$

Theorem 3: Let us assume that $F$ is an icp set-valued mapping at $\lambda_{0} \in A$ with respect to $K_{c}(E)$. Then,

i) if $X^{\lambda} \stackrel{P}{\rightarrow} X^{\lambda} 0$ then $\forall t \in I: \int_{0}^{t} F\left(s, X_{s}^{\lambda}, \lambda\right) d s \stackrel{P}{\rightarrow} \int_{0}^{t} F\left(s, X_{s}^{\lambda_{0}}, \lambda_{0}\right) d s, \lambda \rightarrow \lambda_{0}$,

ii) if for every $A_{1}, A_{2}, \ldots \in K_{c}(E)$ and $\left(\lambda_{n}\right) ; \lambda_{n} \rightarrow \lambda_{0}$ we have

$$
\mathcal{N}\left\{\bigcup_{n \geq 1} F\left(t, A_{n}, \lambda_{n}\right)\right\} \leq k\left(t, \mathcal{N}\left\{\bigcup_{n \geq 1} A_{n}\right\}\right) \text { with } P .1 t \in I \text { a.e., then } X^{\lambda} \stackrel{P}{\rightarrow} X^{\lambda_{0}} \text {. }
$$

Proof: $(i)$ Let $\left(\lambda_{n}\right)$ be an arbitrary sequence convergent to $\lambda_{0}$. Then its every subsequence contains a further subsequence, say, $\left(\lambda_{n}^{\prime}\right)$, such that $X^{\lambda_{n}^{\prime}} \rightarrow X^{\lambda_{0}}$ with $P .1$ in $C_{I}$. Take $\omega$ from an appropriate set (for which this convergence holds). By condition A2, for any $\epsilon>0$, there exists $\delta>0$ such that $H\left(F\left(t, C, \lambda_{n}^{\prime}\right), F\left(t, D, \lambda_{n}^{\prime}\right)\right)<$ $\epsilon / 4 T$, for $n \in N, C, D \in K_{c}(E)$ whenever $H(C, D)<\delta$.

Let $V_{0}$ be an open neighborhood for $\lambda_{0}$ such that 


$$
\text { if } \lambda_{n}^{\prime} \in V_{0} \text { then } \sup _{t \in I} H\left(X_{t}^{\lambda_{n}^{\prime}}, X^{\lambda_{0}}\right)<\delta \text {. }
$$

Let $\left(X_{k}^{\lambda}\right)_{k}$ be a sequence of simple multifunctions (Lemma 1) convergent to $X^{\lambda_{0}}$ for every $t \in I$ and $\omega \in \Omega$. Then for every $t \in I$ and $\lambda \in \Lambda$, we have:

$$
\lim _{k} H\left(F\left(t, \omega, X_{k}^{\lambda_{0}}(t, \omega), \lambda\right), F\left(t, \omega, X_{t}^{\lambda_{0}}(\omega), \lambda\right)=0 \quad P .1\right.
$$

Next by the Lebesgue Dominated Convergence Theorem (via integrably boundedness of $F$ ) we obtain that

$$
\int_{0}^{T} H\left(F\left(s, X_{k}^{\lambda_{0}}(s), \lambda\right), F\left(s, X_{s}^{\lambda_{0}}, \lambda\right)\right) d s \rightarrow 0 P .1
$$

for every $\lambda \in \Lambda$. Hence by (4), after standard calculation we see that

$$
\begin{aligned}
& H\left(\int_{0}^{t} F\left(s, \omega, X_{s}^{\lambda_{n}}(\omega), \lambda_{n}^{\prime}\right) d s, \int_{0}^{t} F\left(s, \omega, X_{s}^{\lambda_{0}}(\omega), \lambda_{0}\right) d s\right) \leq(3 / 4) \epsilon \\
& +H\left(\int_{0}^{t} F\left(s, \omega, X_{k}^{\lambda_{0}}(s, \omega), \lambda_{n}^{\prime}\right) d s, \int_{0}^{t} F\left(s, \omega, X_{k}^{\lambda_{0}}(s, \omega) \lambda_{0}\right) d s\right)
\end{aligned}
$$

for $t \in I, k$ sufficiently large and $\omega$ taken from an appropriate set of probability one.

By Lemma 4, multifunction $F$ is icp at $\lambda_{0}$ with respect to $S(I \times \Omega)$. Hence there exists a sequence $\left(\lambda_{n}^{\prime \prime}\right)$ being a subsequence of $\left(\lambda_{n}^{\prime}\right)$, a subset of $\Omega$ of measure one such that for every $\epsilon>0$ and appropriate $\omega$ we can find an open neighborhood $V_{1}$ of $\lambda_{0}$ with

$$
H\left(\int_{0}^{t} F\left(s, \omega, X_{k}^{\lambda_{0}}(s, \omega), \lambda_{n}^{\prime \prime}\right) d s, \int_{0}^{t} F\left(s, \omega, X_{k}^{\lambda_{0}}(s, \omega), \lambda_{0}\right) d s\right)<\epsilon / 4,
$$

for $t \in I$ and $\lambda_{n}^{\prime \prime} \in V_{1}$. Therefore, taking $n^{\prime \prime}$ sufficiently large and $\lambda_{n}^{\prime \prime} \in V_{0} \cap V_{1}$ we have:

$$
H\left(\int_{0}^{t} F\left(s, \omega, X_{s}^{\lambda_{n}^{\prime \prime}}(\omega), \lambda_{n}^{\prime \prime}\right) d s, \int_{0}^{t} F\left(s, \omega, X_{s}^{\lambda_{0}}(\omega), \lambda_{0}\right) d s\right)<\epsilon
$$

for $t \in I$. This completes the proof of part $(i)$.

Proof of part $(i i)$.

Let $\left(\lambda_{n}\right)$ be a sequence convergent to $\lambda_{0}$. Consider its arbitrary subsequence, denoted for simplicity by the same symbol. We define the multivalued mapping $\Pi: \Omega \rightarrow 2{ }^{C}$ by

$$
\Pi(\omega)=\left\{X \in C_{I}: X^{\lambda_{n}^{\prime}} \rightarrow X \text { in } C_{I} \text { for some sequence }\left(\lambda_{n}^{\prime}\right),\left(\lambda_{n}^{\prime}\right) \subseteq\left(\lambda_{n}\right)\right\} .
$$

By the assumption of the integrably boundness of $F$ it follows that

$$
\forall n \in N, \forall 0 \leq s \leq t \leq T: H\left(X_{t}^{\lambda}, X_{s}^{\lambda}\right) \leq \int_{s}^{t} m(u) d u \text { with P.1. }
$$


Thus the sequence $\left(X^{\lambda} n\right)$ is equicontinuous in $C_{I}$ with P.1. Similarly, (compare [14]) by assumption (iii), it can be proved that $\bigcup_{n \geq 1}\left\{X_{t}^{\lambda}\right\}$ is a relatively compact subset of $E$, for every $t \in I$ with P.1. Thus, by Ascoli Theorem we claim that the sequence $\left(X^{\lambda}\right)$ is relatively compact (with $P .1$ ). Hence the multifunction $\Pi \neq \emptyset P .1$ and has closed values. Moreover, we claim that $\Pi$ is measurable. To see this, let $\Omega_{0}:=\left\{\omega: \Pi(\omega)\right.$ is closed subset of $\left.C_{I}\right\}$. For $X \in C_{I}$ we consider a mapping $\Omega_{0} \ni$ $\omega \rightarrow \operatorname{Dist}(X, \Pi(\omega))$, where $\operatorname{Dist}(X, \Pi(\omega))=\inf _{Y \in \Pi(\omega)} \rho(X, Y)$. Fix $r>0$.

Then $\{\omega: \operatorname{Dist}(X, \Pi(\omega))<r\}=\left\{\omega: \exists Y \in \Pi(\omega): Y \in B_{r}(X)\right\}$, where $B_{r}(X):=$ $\left\{Y \in C_{I}: \rho(X, Y)<r\right\}$. Let $\left\{t_{k}\right\}$ be a sequence of rationals in $I$. Then we get:

$$
\begin{gathered}
\{\omega: \operatorname{dist}(X, \Pi(\omega))<r\}=\left\{\omega: \Pi(\omega) \cap B_{r}(X) \neq \emptyset\right\} \\
=\bigcup_{m \geq 1} \bigcap_{l \geq 1} \bigcup_{j \geq l} \bigcap_{k \geq 1}\left\{\omega: X_{t_{k}}^{\lambda^{n}} j(\omega) \cap B_{r-1 / m}(X) \neq \emptyset\right\} .
\end{gathered}
$$

Since $X_{t_{k}}^{{ }^{\lambda}}{ }_{j}$ is an $\mathscr{F}_{t_{k}}$-measurable multifunction then the last set above belongs to $\sigma$ -

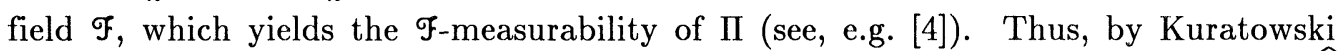
and Ryll-Nardzewski Selection Theorem [10], there exists a measurable selection $X^{\wedge}$ of $\Pi ; X^{\wedge} \in \Pi P .1$. The definition of $\Pi$ implies then that $X^{\lambda_{n}^{\prime}} \rightarrow X^{\wedge} P .1$ in $C_{I}$, for some sequence $\left(\lambda_{n}^{\prime}\right)$ tending to $\lambda_{0}$ and this yields convergence in probability in $C_{I}$. Finally, we claim that $X^{\wedge}$ is a solution of $(I I I)$. Indeed, let us notice that

$$
\begin{gathered}
H\left(\hat{X_{t}}, U+\int_{0}^{t} F\left(s, \hat{X_{s}}, \lambda_{0}\right) d s+\int \sigma_{s} d w_{s}\right) \\
\leq H\left(X_{t}, X_{t}^{\lambda_{n}^{\prime}}\right)+H\left(\int_{0}^{t} F\left(s, X_{s}^{\lambda_{n}^{\prime}}, \lambda_{n}^{\prime}\right) d s, \int_{0}^{t} F\left(s, X_{s}, \lambda_{0}\right) d s\right),
\end{gathered}
$$

with $P .1$ and for $t \in I$.

Since the first term above converges to zero then by $(i)$ the second term converges to zero as well. This completes the proof.

\section{References}

[1] Banaś, J. and Goebel, K., Measures of Noncompactness in Banach Spaces, Marcel Dekker 1980.

[2] Billingsley, P., Probability and Measure, John Wiley, New York 1979.

[3] De Blasi, F.S. and Iervolino, F., Euler method for differential equation with compact, convex valued solutions, Boll. U.M.I 4:4 (1971), 941-949.

[4] Himmelberg, C.J., Measurable relations, Fund. Math. 87 (1975), 53-72.

[5] Himmelberg, C.J. and Van Vleck, F.S., The Hausdorff metric and measurable selections, Topology and its Appl. 20 (1985), 121-133.

[6] Hukuchara, M., Sur 1 application semicontinue dont la valeur est un compact convexe, Funkcial. Ekwac. 10 (1967), 43-66. 
[7] Kisielewicz, M., Differential Inclusions and Optimal Control, Kluwer 1991.

[8] Kisielewicz, M., Method of averaging for differential equation with compact convex valued solutions, Rend. di Matem. 9:3 (1976), 1-12.

[9] Kisielewicz, M., Serafin, B. and Sosulski, W., Existence theorem for functionaldifferential equation with compact convex valued solutions, Demonstratio Math. IX:2 (1976), 229-237.

[10] Kuratowski, K. and Ryll-Nardzewski, Cz., A general theorem on selectors, Bull. Acad. Polon. Sci. Ser, Sci Math. Astronom. Phys. 13 (1965), 397-403.

[11] Michta, M., Set-valued random differential equations in Banach space, Discus. Math. Diff. Inclusions 15:2 (1995), 191-200.

[12] Michta, M., On weak solutions of set-valued random differential equations, Demonstratio Math. 29:3 (1996), 523-528.

[13] Parthasarathy, K.R., Probability Measures on Metric Spaces, Acad. Press, New York 1967.

[14] Tolstonogov, A., Differencjalny je Wkluczenija w Banachowych Prostranstwach, Nauka, Moscow 1986 (Russian).

[15] Wachanija, N.N., Tarieladze, W.I. and Tshobanian, S.A., Werojatnostnyje Raspredelenija w Banachowych Prostranstwach, Nauka, Moscow 1985 (Russian). 


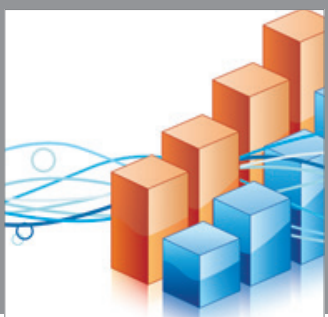

Advances in

Operations Research

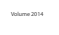

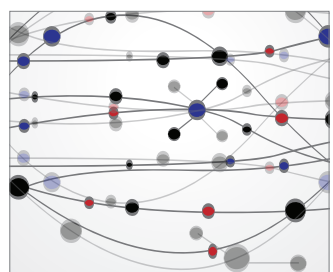

\section{The Scientific} World Journal
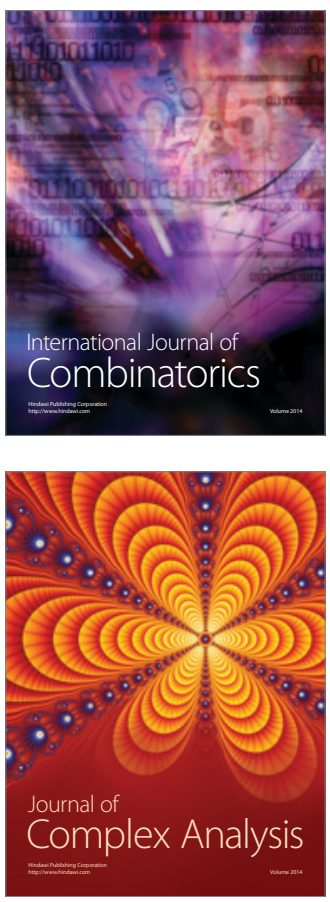

International Journal of

Mathematics and

Mathematical

Sciences
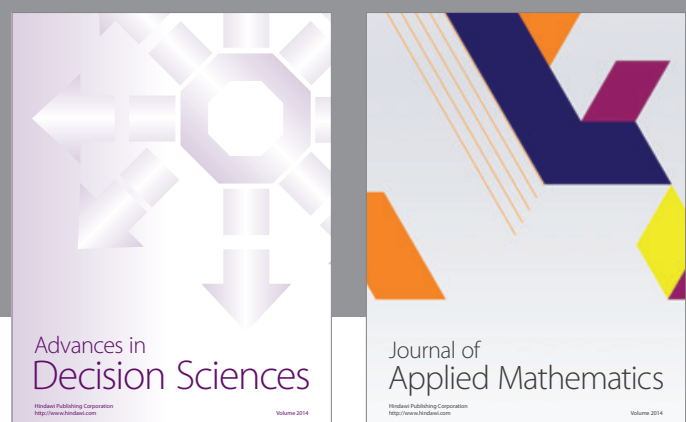

Journal of

Applied Mathematics
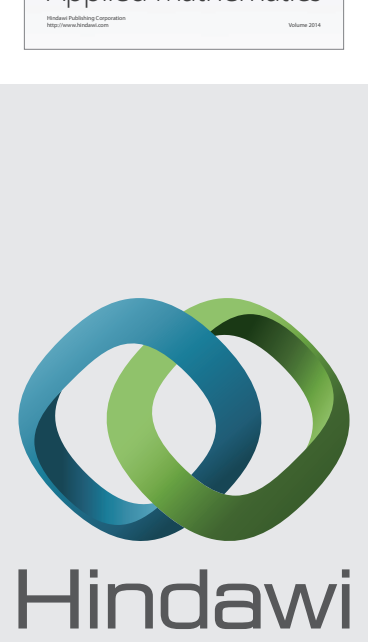

Submit your manuscripts at http://www.hindawi.com
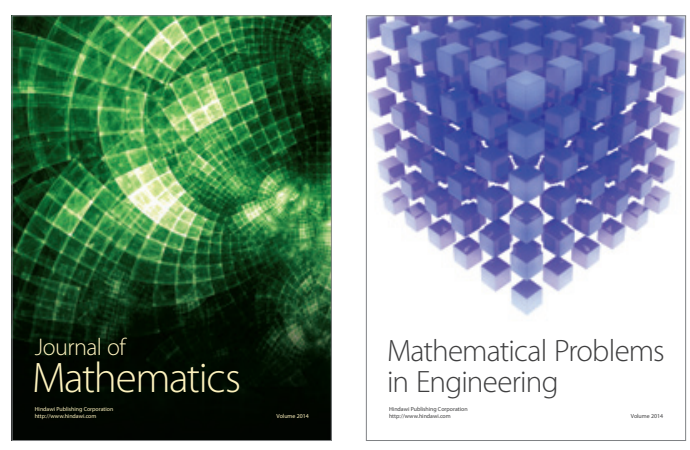

Mathematical Problems in Engineering
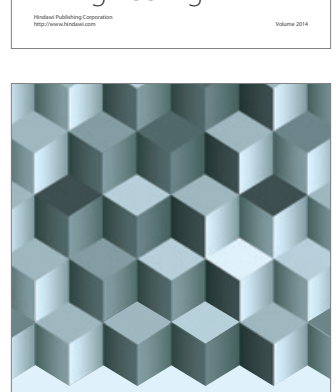

Journal of

Function Spaces
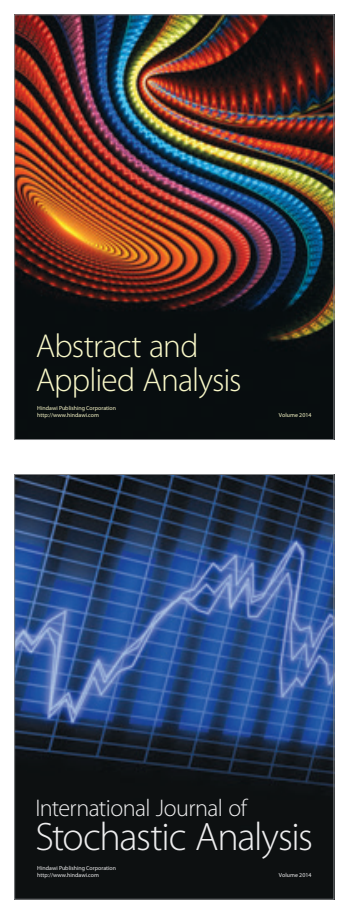

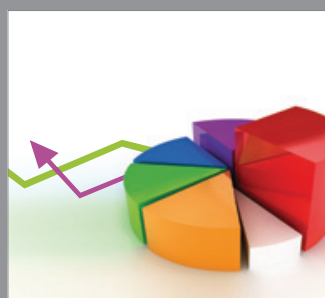

ournal of

Probability and Statistics

Promensencen
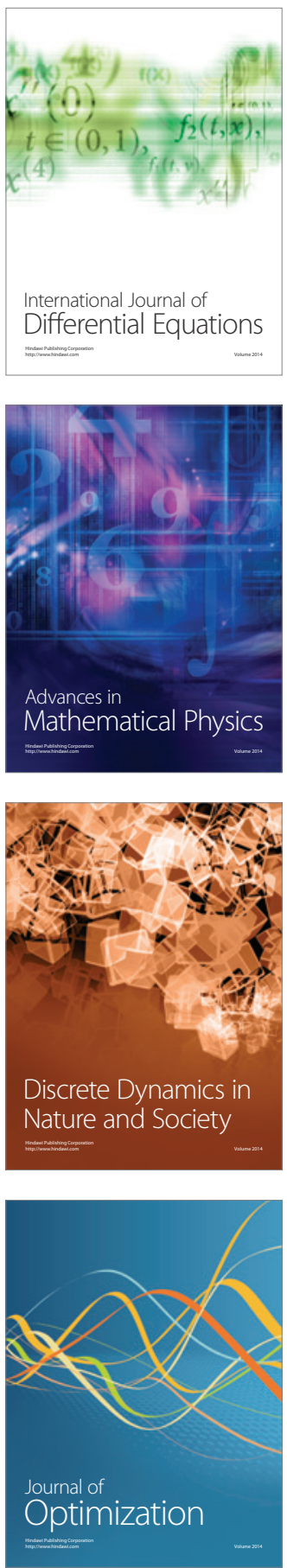